\title{
A DUAL-PATH TEQ STRUCTURE FOR DMT-ADSL SYSTEMS
}

\author{
Ming Ding ${ }^{1}$, Arthur J. Redfern ${ }^{2}$, and Brian L. Evans ${ }^{1}$ \\ ${ }^{1}$ ENS Building, Dept. of Electrical and Computer Engineering, \\ The University of Texas at Austin, Austin, TX 78712-1084 USA \\ email: \{ming,bevans\}@ece.utexas.edu \\ 2 Texas Instruments DSP R\&D Center, Communication Systems Laboratory, \\ 12500 TI Boulevard, MS 8653, Dallas, TX 75243 USA \\ email: redfern@ti.com
}

\begin{abstract}
Discrete Multitone (DMT) modulation is the industry standard for Asymmetrical Digital Subscriber Loop (ADSL) modems. DMT modulation allows for simplified equalization when the effective duration of the channel memory is shorter than the predefined length of the cyclic prefix (CP). A single time-domain equalizer (TEQ) is typically used to truncate the channel memory such that the data rate loss due to the use of the CP is reduced. Because the single TEQ is optimized over all subchannels $\Gamma$ it is difficult to find an ideal solution in which the number of bits supported on each subchannel is optimized. This paper proposes a dual-path TEQ $\Gamma$ which adds a second TEQ that is biased to selected subchannels to increase transmission throughput. The implementation complexity is lower than other band-partition equalization methods such as per tone equalization.
\end{abstract}

\section{INTRODUCTION}

A discrete multitone (DMT) system creates multiple orthogonal subchannels over a broad bandwidth efficiently by using the fast Fourier transform (FFT). An FFT can be efficiently implemented in custom VLSI design or on programmable digital signal processors. DMT has been standardized as the line code for ADSL [1] and is one of two line codes proposed for VDSL [2].

In the ITU standard ADSL system $\Gamma$ a cyclic prefix (CP) is used to eliminate inter-symbol interference (ISI) between successive DMT symbols. The CP is a copy of last $\nu$ samples of the DMT symbol that is prepended to the DMT symbol before transmission. If the channel memory is not longer than the CP length $\Gamma$ then the previous DMT symbol will not interfere with the current DMT symbol. However $\Gamma$ the $\mathrm{CP}$ reduces the channel throughput by a factor of $N /(N+\nu) \Gamma$ where $N$ is the number of samples in the DMT symbol. For downstream transmission $\Gamma N=512$ and $\nu=32 \Gamma$ which results in a data rate loss of $5.88 \%$.

In practice $\Gamma$ the channel memory is usually much longer than $\nu$. Shortening the channel memory can be performed by an FIR filter $\Gamma$ referred to as a time-domain equalizer (TEQ). The TEQ forces the overall impulse response of the cascade of the channel and TEQ to be a desired truncated length $(\nu+1$ samples). Several design methods have been developed for a single TEQ structure $\Gamma$ although the design criteria do not necessarily maximize bit rate.

The Minimum Mean Squared Error (MMSE) [3] method follows from the classical channel memory truncation problem [4] $\Gamma$ in which the cascade of the channel and TEQ approximates a desired target impulse response (TIR). Given the length of TIR $\Gamma$ the TEQ filter coefficients and the TIR itself can be jointly optimized to minimize the MMSE between the TEQ output and the virtual TIR output $\Gamma$ while constraining the energy in the TIR to avoid a trivial solution. The optimum MMSE solution under a unit-norm constraint is the eigenvector corresponding to the minimum eigenvalue of a channel and noise dependent matrix [5]. Derivatives of the MMSE method include the Geometric SNR method [6Г7] and Eigen-approach method [8].

The Maximum Shortening SNR (MSSNR) method is based the observation that residual ISI after the TEQ is caused by the part of the TIR outside of a target window [9]. The method maximizes the Shortening SNR (SSNR) $\Gamma$ which is defined as the ratio of the energy of the TIR inside the target window to the energy outside the target window. The MSSNR method directly minimizes the residual ISI but does not take channel noise into account. The Minimum ISI method generalizes the MSSNR method by adding frequency weighting of the ISI [10]. The frequency weighting takes the subchannel SNRs into account. Both the MSSNR and Minimum ISI methods have been implemented in real time on programmable digital signal processors [10].

The Maximum Bit Rate (MBR) method [10] gives the highest achievable bit rate for a single TEQ structure reported in the open literature [10]. The MBR method develops a new model for subchannel SNR by partitioning the cascade of the channel and single-path TEQ into equivalent signal $\Gamma$ noise $\Gamma$ and ISI paths. Since the MBR method relies on nonlinear optimization $\Gamma$ it is not well suited for fixed-point real-time implementation. The Minimum ISI method mentioned earlier is based on the same subchannel SNR model $\Gamma$ gives near-optimal results $\Gamma$ and is well suited for real-time implementation. In discretized simulation $\Gamma \mathrm{a}$ three-tap Minimum ISI TEQ can generally achieve the same bit rate as a 32-tap MMSE TEQ [10].

An alternate receiver structure $\Gamma$ per tone equalization $[11] \Gamma$ uses a multitap frequency-domain equalizer (FEQ) 
structure to replace the conventional TEQ and single-tap FEQ structure. Using 32-tap per tone equalization improves the achievable bit rate by roughly $8.3 \%$ when compared to a single-path 32-tap MMSE TEQ for 26 AWG loops using a discretized simulation [12]. During data transmission $\Gamma$ per tone equalization increases computational complexity and memory usage by a factor of 2 and 10Гrespectively $\Gamma$ when compared a single-path 32-tap MMSE TEQ. During training $\Gamma$ per tone equalization requires substantially more computational complexity and memory usage to achieve the bit rate increase.

In this paper $\Gamma$ we develop a dual-path TEQ structure to the perform channel truncation for DMT transceivers. Section 2 explores the performance gap between using a single TEQ and per tone equalization. Section 3 proposes a dual-path TEQ structure to narrow the gap. Section 4 provides simulation examples to demonstrate that the dualpath TEQ bit rate results are comparable to per tone equalization but at a much lower implementation cost.

\section{MOTIVATION FOR DUAL-PATH TEQ}

In this section $\Gamma$ we motivate the dual-path TEQ architecture. Let $t$ indicate the DMT symbol index and $n=0,1, \ldots$, $N+\nu-1$ indicate the samples within the given symbol plus the CP. The TEQ output is given as

$$
z_{t}(n)=\sum_{\tau=0}^{L_{w}} w(\tau) y_{t}(n-\tau)
$$

where $w(\tau)$ denotes the $\tau$ th coefficient of the length $L_{w}+1$ TEQ and $y_{t}(\cdot)$ is the received sequence. The TEQ operations in receiver can be written in matrix form as

$$
\begin{aligned}
\mathbf{z}_{t}= & {\left[\begin{array}{cccc}
y_{t}(\nu) & y_{t}(\nu-1) & \cdots & y_{t}\left(\nu-L_{w}\right) \\
y_{t}(\nu+1) & y_{t}(\nu) & \cdots & y_{t}\left(\nu-L_{w}+1\right) \\
\vdots & \vdots & \ddots & \vdots \\
y_{t}(N+\nu-1) & \cdots & \cdots & y_{t}\left(N+\nu-1-L_{w}\right)
\end{array}\right] } \\
& \times\left[\begin{array}{c}
w(0) \\
w(1) \\
\vdots \\
w\left(L_{w}\right)
\end{array}\right] \\
= & \mathbf{Y}_{t} \mathbf{w}
\end{aligned}
$$

where $\mathbf{Y}_{t}$ is a Toeplitz matrix which contains the received signal for detection of $t$ th symbol.

The CP-removed TEQ output is then transferred back to frequency domain by means of an FFT. To recover the transmitted datara one-tap FEQ is applied for each tone of the FFT output to undo the equalized channel attenuation. Let $\mathbf{F}$ denote the $N \times N$ DFT matrix and the diagonal matrix $\mathbf{G}$ denote the FEQ matrix with the $i$ th diagonal entry corresponding to the complex one-tap FEQ for the $i$ th tone. We express the estimated DMT symbol as

$$
\begin{aligned}
\tilde{\mathbf{x}}_{\mathbf{t}} & =\left[\begin{array}{c}
\tilde{x}_{t}(0) \\
\tilde{x}_{t}(1) \\
\vdots \\
\tilde{x}_{t}(N-1)
\end{array}\right] \\
& =\left[\begin{array}{cccc}
g(0) & g(1) & & \\
& & \ddots & \\
& & & g(N-1)
\end{array}\right]\left[\begin{array}{c}
\mathbf{f}^{T}(0) \\
\mathbf{f}^{T}(1) \\
\vdots \\
\mathbf{f}^{T}(N-1)
\end{array}\right] \mathbf{Y}_{t} \mathbf{w} \\
& =\mathbf{G F Y}_{t} \mathbf{w}
\end{aligned}
$$

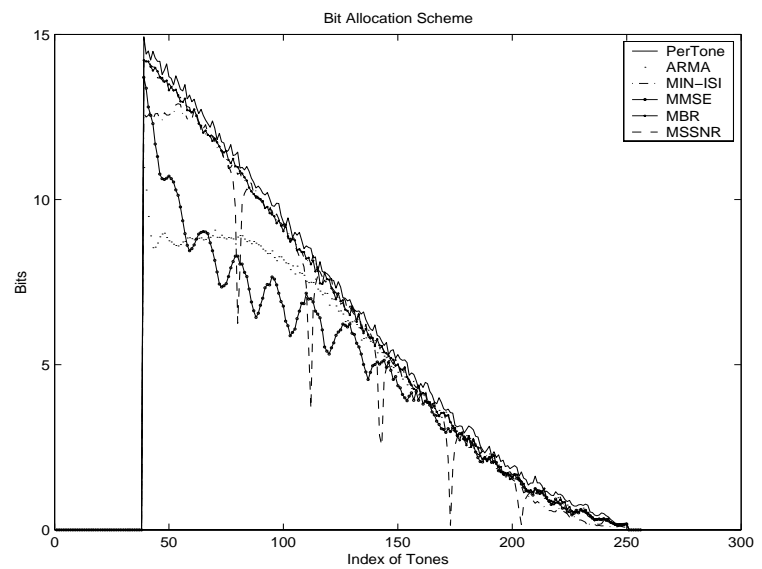

Figure 1: Sample bit allocation schemes for simulated channel with Additive White Gaussian (AWGN) noise.

where $\mathbf{f}^{T}(i)$ denotes the $i$ th row of the DFT-matrix.

Using (3) $\Gamma$ we write the estimated data for tone $i$ as

$$
\tilde{x}_{t}(i)=g(i) \mathbf{f}^{T}(i) \mathbf{Y}_{t} \mathbf{w}=g(i) \mathbf{s}_{t}^{T}(i) \mathbf{w}
$$

where $\mathbf{s}_{t}(i)=\mathbf{f}^{T}(i) \mathbf{Y}_{t}$. By moving $g(i)$ to the $\operatorname{right} \Gamma$ we have a complex $\left(L_{w}+1\right)$-tap FEQ

$$
\tilde{x}_{t}(i)=g(i) \mathbf{s}_{t}^{T}(i) \mathbf{w}=\mathbf{s}_{t}^{T}(i) \mathbf{p}(i)
$$

where $\mathbf{p}(i)$ is the $\left(L_{w}+1\right)$-tap FEQ for the $i$ th tone. Equation $(5)$ is the foundation for per tone equalization.

The form of the per tone equalizer coefficients does not need to be restricted to real filter coefficients multiplied by a complex scalar. Instead $\Gamma$ the equalizer can have complex coefficients. Due to the extra freedom in the per tone equalization structure it generally performs better than the TEQ-FEQ structures in bit rate.

Fig. 1 shows sample bit allocation schemes for transmission over the AWG 26 channel. The simulated channel is a simplified discretized model of a real channel; no analog processing by the transceiver is included. In this case $\Gamma$ ISI and additive noise are the major impairments. We design the per tone equalizer using a least-squares method.

Based on simulations $\Gamma$ we make the following observations on the performance of per tone equalization vs. that of single TEQ structures:

1. Per tone equalization not only achieves higher aggregate bit rates $\Gamma$ but also benchmarks Achievable Bit Rate (ABR) for any single tone.

2. The performance gap tends to be larger in favor of per tone equalization as the transmission environment becomes more sophisticated.

3. The performance gap for any single tone is not universally wide. In tones associated with higher SNR the improvement of per tone tends to be significant. For other tones $\Gamma$ the improvement is not significant. 


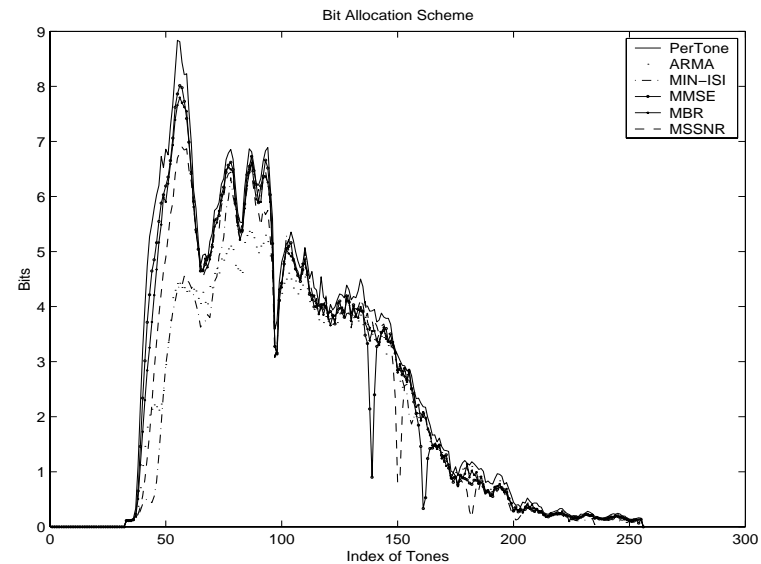

Figure 2: Sample bit allocation schemes for ANSI loop 13 with NEXT.

Fig. 2 shows bit allocation schemes for an ADSL system over ANSI loop 13 with NEXT from 24 DSL disturbers to justify the above observations. Based on the above observations $\Gamma$ especially observation $3 \Gamma$ it is not necessary to use a separate TEQ for each single tone. Alternately $\mathrm{Twe}$ propose to use a second TEQ whose goal is to optimize those subchannels with the best chance of improving the bit rate. These subchannels with the potential for the best SNR are known before equalizer training because they can be estimated from Reverb (a periodic sequence $\Gamma$ which thus needs no TEQ) which occurs earlier in training.

\section{DESIGN OF DUAL-PATH TEQ}

A dual-path TEQ structure for the DMT receiver passes the received data through two paths instead of one path. One possible approach is to give each path its own TEQ FFT and one-tap FEQs. The FEQ outputs would be fed

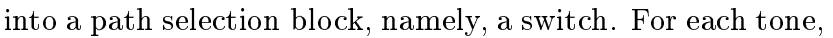
we choose output from one of the two paths according to a preset rule. The rule should be set during initialization stage. A possible criterion could be that we choose the path with a higher subchannel SNR after FEQ for each tone. In other words $\Gamma$ the FEQ output for each tone $i$ could be

$$
\tilde{x}_{i}= \begin{cases}\tilde{x}_{i 1} & \text { if } \mathrm{SNR}_{i 1}>\mathrm{SNR}_{i 2} \\ \tilde{x}_{i 2} & \text { otherwise }\end{cases}
$$

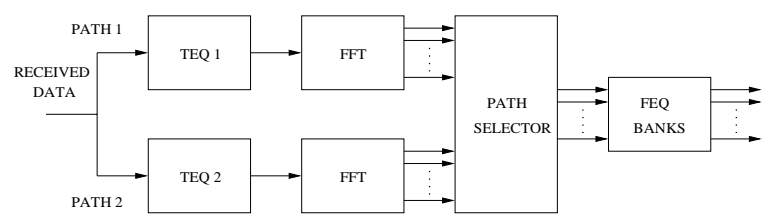

Figure 3: Structure of a dual-path TEQ.
In Fig. 3Гwe simplify this dual-path TEQ structure to move the path selector before the one-tap FEQ. This modification works well provided that a well-defined metric after the FFT was available. In this case only one set of FEQs are implemented in the receiver.

One choice is that each TEQ optimizes a different part of the bandwidth and the subchannels of interest span the entire bandwidth. However $\Gamma$ such a partition is difficult to optimize. We choose a slightly different approach as follows:

1. Path 1 is a path with normal TEQ $\Gamma$ which optimizes the entire bandwidth.

2. Path 2 exploits a second TEQ $\Gamma$ which optimizes the subchannels within a preset window of frequencies.

The subchannels with higher SNR as seen in Figs. 1 and 2 generally have more room for bit rate improvement via per tone equalization. Accordingly $\Gamma$ these subchannels should be put into the window. A simple subchannel selection method is to slide a window through all subchannels $\Gamma$ and the desired start tone index $i$ can be determined by

$$
i_{\text {start }}=\arg \max _{i} \sum_{k=i}^{k=i+W L-1} S_{x, i}\left|H_{i}\right|^{2} / S_{n, i}
$$

where $W L$ is the window length $\Gamma$ and $S_{x, i} \Gamma S_{n, i}$ and $H_{i}$ are the transmitted signal power $\Gamma$ channel noise power $\Gamma$ and frequency response for the $i$ th toneTrespectively.

Any existing algorithm can be used for training the first TEQ. However Tonly a few TEQ design methods that have some control over the TEQ frequency response are good candidates for designing the second TEQ. Among them $\Gamma$ MBR and Minimum ISI methods are well suited for considering the ABR for selected subchannels.

The basic idea of the MBR and Minimum ISI methods is to decompose the TEQ output $h(n) * w(n)$ into the desired part and ISI corrupted part by windowing

$$
g(n)= \begin{cases}1 & \text { if } \Delta \leq n \leq \Delta+\nu \\ 0 & \text { otherwise }\end{cases}
$$

where $\Delta$ is the delay. The TEQ output is then written as

$$
\begin{aligned}
y(n)= & h(n) * w(n) * x(n)+w(n) * \nu(n) \\
= & h^{\text {signal }}(n) * x(n)+h^{I S I}(n) * x(n) \\
& +w(n) * \nu(n)
\end{aligned}
$$

For each tone $\mathrm{Ta}$ following definition of SNR can be formed

$$
\mathrm{SNR}_{i}=\frac{\left|H_{i}^{\text {signal }}\right|^{2} S_{x, i}}{\left|H_{i}^{I S I}\right|^{2} S_{x, i}+\left|W_{i}\right|^{2} S_{n, i}}
$$

where $H_{i}^{\text {signal }} \Gamma_{i}^{I S I}$ and $W_{i}$ are $i$ th DFT sample of $h^{\text {singal }} \Gamma$ $h^{I S I}$ and wTrespectively. We write (10) in matrix form as

$$
\mathrm{SNR}_{i}=\frac{\left|\mathbf{q}_{i}^{H} \mathbf{G H} \mathbf{w}\right|^{2} S_{x, i}}{\left|\mathbf{q}_{i}^{H} \mathbf{D H}\right|^{2} S_{x, i}+\left|\mathbf{q}_{i}^{H} \mathbf{F H w}\right|^{2} S_{n, i}}
$$

where

$$
\begin{aligned}
\mathbf{G} & =\operatorname{diag}[g(0) g(1) \ldots g(N-1)]^{T} \\
\mathbf{D} & =\mathbf{I}-\mathbf{G}
\end{aligned}
$$




$$
\begin{aligned}
\mathbf{H} & =\left[\begin{array}{cccc}
h(0) & h(-1) & \ldots & h\left(-\left(L_{w}-1\right)\right) \\
h(1) & h(0) & \ldots & h\left(-\left(L_{w}-2\right)\right) \\
\vdots & \vdots & \ddots & \vdots \\
h(N-1) & h(N-2) & \ldots & h\left(-\left(N-L_{w}\right)\right)
\end{array}\right] \\
\mathbf{q}_{i} & =\left[\begin{array}{lllll}
1 & e^{j 2 \pi i / N} & \ldots & e^{j 2 \pi i(N-1) / N}
\end{array}\right]^{T}
\end{aligned}
$$

Here $\Gamma \mathbf{q}_{i}^{H} \mathbf{F H w}$ is the $i$ th N-point FFT coefficient of $\mathbf{w}$.

The MBR method is then performed as a nonlinear optimization to maximize the bit allocation function

$$
b_{\mathrm{DMT}}=\sum_{i} \log _{2}\left(1+\frac{\mathrm{SNR}_{i}}{\Gamma}\right)
$$

where $\Gamma$ is the SNR gap [3]. If the ISI term in (10) could be forced to be zeroГthen the subchannel SNR would not depend on the equalizer settings [10]. It becomes the highest achievable SNR $S_{x, i}\left|H_{i}\right|^{2} / S_{n, i}$. The Minimum ISI method tries to minimize the total distortion power of all used subchannels given by

$$
\mathbf{w}^{T} \mathbf{H}^{T} \mathbf{D}^{T} \sum_{i}\left(\mathbf{q}_{i}\left|H_{i}\right|^{2} \frac{S_{x, i}}{S_{n, i}} \mathbf{q}_{i}^{H}\right) \mathbf{D H w}=\mathbf{w}^{T} \mathbf{X} \mathbf{w}
$$

To prevent minimization of the signal power 5 we put a constraint on the effective signal energy:

$$
\left|h^{\text {signal }}\right|^{2}=\mathbf{w}^{T} \mathbf{H}^{T} \mathbf{G}^{T} \mathbf{G H} \mathbf{H}=\mathbf{w}^{T} \mathbf{Y} \mathbf{w}=1
$$

Here we convert the TEQ design problem to a constrained minimization problem. The optimal solution is one of the generalized eigenvectors of the matrix pencil $(\mathbf{X}, \mathbf{Y})$.

\section{SIMULATIONS}

Fig. 4 shows the resulting bit allocation for a dual-path TEQ ADSL system over ANSI loop 13 with NEXT from 24 DSL disturbers. The MBR method is used to train the TEQs. The final bit allocation scheme would be the outer envelop of the two overlapped curves. While the first TEQ is an optimum solution for all used tones $\Gamma$ the second TEQ optimizes tones 55-85 only. Fig. 4 shows that for some tones with higher SNR $\mathrm{especially \Gamma} \mathrm{those} \mathrm{with} \mathrm{peaks} \Gamma$ the second TEQ performs better. For other tones $\Gamma$ the second TEQ has a deep notch. The dual-path TEQ increased the ABR by $4 \%$ from 2.508 Mbps to 2.602 Mbps.

\section{CONCLUSIONS}

Using a dual-path TEQ results in a higher achievable bit rate than a single-path TEQ $\Gamma$ if an appropriate design technique is available. The second TEQ used for equalization of selected subchannels can be designed in the time domain or $\Gamma$ more naturally $\Gamma$ the frequency domain. A good frequencydomain approach for this optimization problem is currently under consideration.

\section{ACKNOWLEDGMENTS}

The authors would like to thank Nirmal Warke and Murtaza Ali at the Texas Instruments DSPS R\&D Center for their valuable support $\Gamma$ conversations $\Gamma$ and feedback.

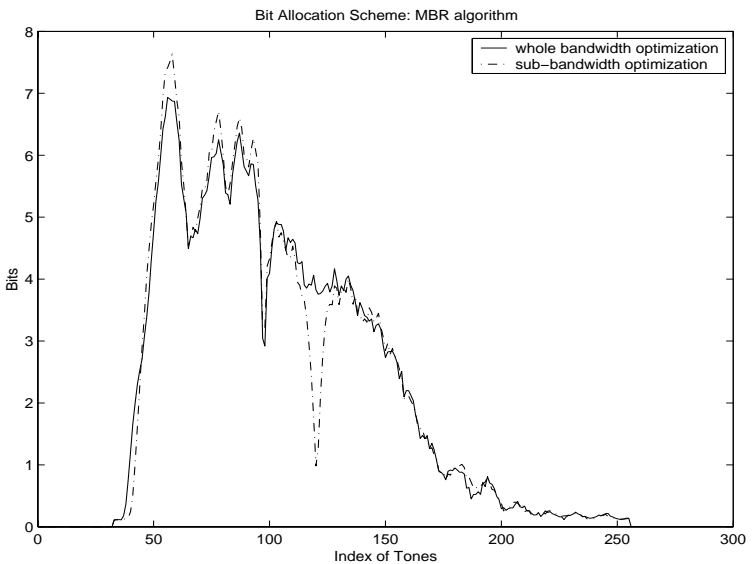

Figure 4: Bit allocation scheme for a dual-path TEQ.

\section{REFERENCES}

[1] W. Y. Chen, DSL Simulation Techniques and Standards Development for Digital Subscriber Line Systems, McMillan Technical Publishing, Indianapolis, Indiana, 1998.

[2] V. Oksman, "Standard VDSL technology," IEEE 802.3 Standards Group, July 2001, http://www.ieee802.org/ 3/efm/public/jul01/presentations/oksman_1_0701.pdf.

[3] J. S. Chow and J. M. Cioffi, "A cost-effective maximum likelihood receiver for multicarrier systems," in Proc. IEEE Int. Conf. Comm., June 1992, vol. 2, pp. 948-952.

[4] D. D. Falconer and F. R. Magee, "Adaptive channel memory truncation for maximum likelihood sequence estimation," Bell System Technical J., vol. 52, no. 9, pp. 1541-1562, Nov. 1973.

[5] N. Al-Dhahir and J. M. Cioffi, "Efficiently computed reduced-parameter input-aided MMSE equalizers for ML detection: A unified approach," IEEE Trans. on Info. Theory, vol. 42, no. 3, pp. 903-915, May 1996.

[6] N. Al-Dhahir and J. M. Cioffi, "Optimum finite-length equalization for multicarrier transceivers," IEEE Trans. on Comm., vol. 44, no. 1, pp. 56-63, Jan. 1996.

[7] N. Al-Dhahir and J. M. Cioffi, "A band-optimized reducedcomplexity equalized multicarrier transceiver," IEEE Trans. on Comm., vol. 45, no. 8, pp. 948-956, Aug. 1997.

[8] B. Farhang-Bouroujeny and M. Ding, "Design methods for time-domain equalizers in DMT transceivers," IEEE Trans. on Comm., vol. 49, no. 3, pp. 554-562, Mar. 2001.

[9] P. J. W. Melsa, R. C. Younce, and C. E. Rohrs, "Impulse response shortening for discrete multitone transceivers," IEEE Trans. on Comm., vol. 44, no. 12, pp. 1662-1672, Dec. 1996.

[10] G. Arslan, B. L. Evans, and S. Kiaei, "Equalization for discrete multitone transceivers to maximize bit rate," IEEE Trans. on Signal Processing, vol. 49, no. 12, Dec. 2001.

[11] K. Van Acker, G. Leus, M. Moonen, O. van de Wiel, and T. Pollet, "Per tone equalization for DMT-based systems," IEEE Trans. on Comm., vol. 49, no. 1, pp. 109-119, Jan. 2001.

[12] K. Van Acker, Equalization and Echo Cancellation for DMT-Based DSL Modems, Katholieke Universiteit Leuven, Belgium, 2001. 